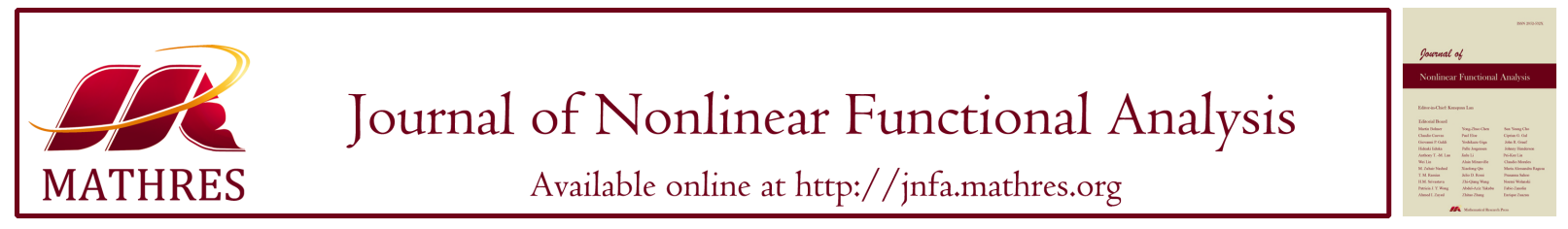

\title{
SYNCHRONIZATION ON THE NON-AUTONOMOUS CELLULAR NEURAL NETWORKS WITH TIME DELAYS
}

\author{
AZHAR HALIK ${ }^{1}$, AISHAN WUMAIER ${ }^{2, *}$ \\ ${ }^{1}$ College of Mathematics and System Sciences, Xinjiang University, Urumqi 830046, China \\ ${ }^{2}$ College of Information Science and Engineering, Xinjiang University, Urumqi 830046, China
}

\begin{abstract}
This paper is concerned with a general decay synchronization (GDS) between two delayed non-autonomous cellular neural networks. A non-autonomous case and infinite delays are taken into consideration. By using the Lyapunov stability theory and employing useful inequality techniques, some sufficient conditions on the GDS of the considered system are established based on a type of nonlinear control. In addition, an example with numerical simulations is provided to demonstrate the effectiveness and feasibility of the obtained results.
\end{abstract}

Keywords. Cellular neural networks; General decay synchronization; Time-varying coefficients; Time delays.

\section{INTRODUCTION}

In the past few decades, the dynamical properties of neural networks (NNs) including stability, synchronization, bifurcation, periodic attractors, and chaotic attractors have attracted considerable attention since they have been successfully applied in many areas, such as, image processing, pattern recognition, associative memory, and optimization problems; see, e.g., [1]-[8] and the references therein. In particular, the research on the synchronization of NNs has arrested much attention ([9]-[15]) as there are many benefits of having synchronization or chaos synchronization in some engineering applications, such as, secure communication, information science, harmonic oscillation generation, language emergence and development [3]. In addition, the synchronization, as a typical collective behavior, has been observed in biological systems, such as, synchronous fireflies, swarming of fishes, flocking of birds [4]. Therefore, it is important to investigate the synchronization behaviors in neural networks.

It is well known that one of the central issues of the synchronization studies in neural networks is how to achieve the synchronization between drive-response systems. However, when

${ }^{*}$ Corresponding author.

E-mail addresses: azhar1117@163.com (A. Halik), hasan1479@xju.edu.cn (A. Wumaier).

Received July 5, 2020; Accepted December 6, 2020.

(C)2020 Journal of Nonlinear Functional Analysis 
we investigate the synchronization behaviors of the NNs, we should introduce time delay into the model foundation due to the fact that the time delay is inevitable and will occur during the signal transmission among the neurons, which will affect the stability of the neural system and may lead to some complex dynamic behaviors such as periodic oscillation, bifurcation, or chaos [5]. In recent years, as an important neural network model, the delayed neural networks (DNNs) have been studied in many books and papers (see, e.g., [16]-[25] and the references therein). Also, the synchronization study of delayed neural networks (DNNs) has attracted considerable attention of many researchers from various fields, and many important results were established. In these studies, many methods were exploited, such as, the Lyapunov function approach [1, 2], linear matrix inequality [3], matrix measure strategy and Lyapunov approach [4], Lyapunov theory and fractional-order differential inequalities [6], and generalized Halanay inequalities and matrix measure approaches [7]. And, several control strategies, such as, sampled-data control[1, 8, 9, 10], linear feedback control[2], pinning control[4, 11], impulsive control[12] and fuzzy control[13] are widely applied to controlling and synchronizing the DNNs.

In general, there are two types of neural networks in recent studies. The first type is autonomous NNs and the second type is non-autonomous NNs. The main difference between the autonomous NNs and the non-autonomous NNs is that the intrinsic parameters of autonomous NNs are constants and has no input effect. But the intrinsic parameters of the non-autonomous NNs are variables and have input effect. Up to now, the majority of existing results are devoted to the autonomous NNs [12]-[26], and there are few papers considered the non-autonomous NNs $[27,28]$. In $[27,28]$, the authors considered the following non-autonomous cellular neural networks(CNNs) with time variable delays and infinite delays

$$
\begin{aligned}
\dot{x}_{i}(t)= & -c_{i}(t) x_{i}(t)+\sum_{j=1}^{n} a_{i j}(t) f_{j}\left(x_{j}\left(t-\tau_{i j}(t)\right)\right) \\
& +\sum_{j=1}^{n} b_{i j}(t) \int_{0}^{\infty} K_{i j}(u) g_{j}\left(x_{j}(t-u)\right) d u+I_{i}(t),
\end{aligned}
$$

The authors obtained some sufficient conditions on the exponential convergence for system (1.1) by using the differential inequality strategies. But, in [27, 28], the authors did not consider the estimation of exponential convergent rate. On the other hand, in the process of investigating neural networks, the estimate of the convergent rate of the synchronization is one of the major concerns and useful for studying the synchronization of chaotic systems [22]. However, in some cases, the convergence rate of the synchronization can not be obtained or it is very difficult to estimate. For example, consider the following equation [23]

$$
\dot{x}(t)=-\frac{1}{2} x^{3}, \quad t \geq 0 .
$$

Although, we can find that the above equation is asymptotically stable, we can not be able to estimate the convergent rate of its solution. Recently, a new concept of the synchronization, namely, the general decay synchronization (GDS) was introduced for a class of chaotic NNs by Wang, Shen and Zhang [24, 25] and this GDS can deal with the above mentioned problem. There has been some literatures related to the study of the general decay synchronization for neural networks with time delays [21]-[26]. However, these models in [21]-[26] are all autonomous and without infinite delays. Besides, there is no theoretical result published on 
the research on the general decay synchronization for delayed cellular neural networks with time-varying coefficients and infinite delays.

Motive and inspired by the above results, in this paper, we extend system (1.1) to the more general case and investigate the following non-autonomous CNNs system with time variable delays and infinite delays

$$
\begin{aligned}
\dot{x}_{i}(t)= & -c_{i}(t) x_{i}(t)+\sum_{j=1}^{n} d_{i j}(t) f_{j}\left(x_{j}(t)\right)+\sum_{j=1}^{n} a_{i j}(t) f_{j}\left(x_{j}\left(t-\tau_{i j}(t)\right)\right) \\
& +\sum_{j=1}^{n} b_{i j}(t) \int_{0}^{\infty} K_{i j}(u) g_{j}\left(x_{j}(t-u)\right) d u+I_{i}(t) .
\end{aligned}
$$

The main purpose of this paper is to establish some sufficient conditions on the general decay synchronization for system (1.2). The method used in this paper is mainly motivated by the work done by Wang, Shen and Zhang [24, 25] and the Lyapunov-Krasovskii function method.

The main contributions of this paper are:

1) The general non-autonomous CNNs system with time variable delays and infinite delays is considered.

2) Compared with previous related results, we, for the first time, consider the GDS problem for non-autonomous CNNs system with time variable delays and infinite delays, and derive some sufficient conditions, which guarantee the considered system to achieve GDS. In addition, we are able to estimate the convergence rate of the synchronization by GDS.

3) Since the general decay synchronization may be specialized as exponential synchronization, polynomial synchronization and logarithmic synchronization, the results presented in this paper can be seen as the improvement and extension of the previously known results.

\section{PRELIMinaries}

In system (1.2), $i \in \Pi \triangleq\{1,2, \cdots, n\}, n$ corresponds to the number of units in a neural network; $x_{i}(t)$ denotes the state vector of the $i$ th unit at the time $t ; c_{i}(t)>0$ denotes the rate with which the $i$ th unit will reset its potential to the resting state in isolation when disconnected from the network and external inputs at the time $t ; a_{i j}(t), b_{i j}(t)$ and $d_{i j}(t)$ denote the connection weights between the $i$ th neuron and the $j$ th neuron at time $t ; f_{i}(\cdot)$ and $g_{i}(\cdot)$ are the nonlinear activation functions of signal transmission; $I_{i}(t)$ denotes the external bias on the $i$ th unit at the time $t ; K_{i j}(u)$ corresponds to the transmission delay kernel satisfying $\int_{0}^{\infty} K_{i j}(s) d u=1$, and $\tau_{i j}(t)$ denotes the transmission time-varying delays of the $i$ th unit along the axon of the $j$ th unit at the time $t$ satisfying $0 \leq \tau_{i j}(t) \leq \tau_{i j}$.

In this paper, we always use $\Pi \triangleq\{1,2, \cdots, n\}$ and $R^{+}=[0,+\infty)$, unless otherwise stated. The initial conditions associated with system (1.2) are given by

$$
x_{i}(s)=\varphi_{i}(s), s \in(-\infty, 0], i=1,2, \cdots, n,
$$

where $\varphi(s)=\left(\varphi_{1}(s), \varphi_{2}(s), \cdots, \varphi_{n}(s)\right) \in C\left((-\infty, 0], R^{n}\right)$ denotes the Banach space of all continuous functions mapping $(-\infty, 0]$ into $R^{n}$ with norm defined by

$$
\|\varphi\|=\sup _{s \in[-\tau, 0]}\|\varphi(s)\|
$$

with $\|\varphi(s)\|=\max _{i \in \Pi}\left|\varphi_{i}(s)\right|$. 
For a continuous and bounded function $f(t)$, we define

$$
f^{-}=\inf _{t \in R}\{f(t)\}
$$

and

$$
f^{+}=\sup _{t \in R}\{f(t)\} .
$$

Throughout this paper, we assume that the following assumptions are satisfied.

$\mathbf{H}_{1}$ Activation functions $f_{j}(u)$ and $g_{j}(u)$ are continuous and there exist nonnegative constants $H_{j}, K_{j}, M_{j}, O_{j} \geq 0$ such that, for any $v_{1}, v_{2} \in R$,

$$
\left|f_{j}\left(v_{1}\right)-f_{j}\left(v_{2}\right)\right| \leq H_{j}\left|v_{1}-v_{2}\right|+M_{j},\left|g_{j}\left(v_{1}\right)-g_{j}\left(v_{2}\right)\right| \leq K_{j}\left|v_{1}-v_{2}\right|+O_{j} .
$$

$\mathbf{H}_{2}$ Time-varying delay $\tau_{i j}(t)$ is differentiable, and there exists a real number $0 \leq \zeta_{i j} \leq 1$ such that, for any $t \in R^{+}$,

$$
0 \leq \dot{\tau}_{i j}(t) \leq \zeta_{i j}
$$

In this paper, we consider system (1.2) as the drive system, and the response system is given as follows

$$
\begin{aligned}
\dot{y}_{i}(t)= & -c_{i}(t) y_{i}(t)+\sum_{j=1}^{n} d_{i j}(t) f_{j}\left(y_{j}(t)\right)+\sum_{j=1}^{n} a_{i j}(t) f_{j}\left(y_{j}\left(t-\tau_{i j}(t)\right)\right) \\
& +\sum_{j=1}^{n} b_{i j}(t) \int_{0}^{\infty} K_{i j}(u) g_{j}\left(y_{j}(t-u)\right) d u+I_{i}(t)+u_{i}(t),
\end{aligned}
$$

where $u_{i}(t)$ is the controller to be designed.

Let $e_{i}(t)=y_{i}(t)-x_{i}(t)$. From (1.2) and (2.1), the error dynamical system is expressed as

$$
\begin{aligned}
\dot{e}_{i}(t)= & -c_{i}(t) e_{i}(t)+\sum_{j=1}^{n} d_{i j}(t) \widetilde{f}_{j}\left(e_{j}(t)\right)+\sum_{j=1}^{n} a_{i j}(t) \widetilde{f}_{j}\left(e_{j}\left(t-\tau_{i j}(t)\right)\right) \\
& +\sum_{j=1}^{n} b_{i j}(t) \int_{0}^{\infty} K_{i j}(u) \widetilde{g}_{j}\left(e_{j}(t-u)\right) d u+u_{i}(t),
\end{aligned}
$$

where $\tilde{f}_{j}\left(e_{j}(t)\right)=f_{j}\left(y_{j}(t)\right)-f_{j}\left(x_{j}(t)\right)$.

Now, we present the definitions of the $\psi$-type function and the GDS.

Definition 2.1. $[24,25]$ A function $\psi: R^{+} \rightarrow[1,+\infty)$ is said to be $\psi$-type function if it satisfies the following conditions

1) It is differentiable and nondecreasing;

2) $\psi(0)=1$ and $\psi(+\infty)=+\infty$;

3) $\tilde{\psi}(t)=\dot{\psi}(t) / \psi(t)$ is nondecreasing and $\psi^{*}=\sup _{t \geq 0} \tilde{\psi}(t)<+\infty$, where $\dot{\psi}(t)$ is the time derivative of $\psi(t)$;

4) For any $t, s \geq 0, \psi(t+s) \leq \psi(t) \psi(s)$.

It is not difficult to check that $\psi(t)=e^{\alpha t}$ and $\psi(t)=(1+t)^{\alpha}$, for any $\alpha>0$, satisfy the above four conditions. Thus, they can be seen as $\psi$-type functions. 
Definition 2.2. [24, 25] The drive-response systems (1.2) and (2.1) are said to be general decay synchronized if there exist a constant $\varepsilon>0$ and a $\psi$-type function $\psi$ such that, for any solutions $x(t)=\left(x_{1}(t), x_{2}(t), \cdots, x_{n}(t)\right)$ of system (1.2) and $y(t)=\left(y_{1}(t), y_{2}(t), \cdots, y_{n}(t)\right)$ of system $(2.1)$,

$$
\limsup _{t \rightarrow+\infty} \frac{\log \|y(t)-x(t)\|}{\log \psi(t)} \leq-\varepsilon
$$

where $\varepsilon>0$ can be seen as the convergence rate as synchronization error approaches zero.

$\mathbf{H}_{3}$ : For the function $\psi(t)$ given in Definition 2.1, there exist a function $\rho(t) \in C\left(R, R^{+}\right)$and a constant $\delta$ such that, for any $t \geq 0$,

$$
\tilde{\psi}(t) \leq 1, \quad \sup _{t \in[0,+\infty)} \int_{0}^{t} \psi^{\delta}(s) \rho(s) d s<+\infty .
$$

Now, we present a useful lemma, which is essential to our later study.

Lemma 2.3. $[24,25]$ Under assumption $\mathbf{H}_{3}$, assume that the synchronization error $e(t)=y(t)-$ $x(t)$ of driver-response systems (1.2) and (2.1) satisfies the differential equation $\dot{e}(t)=g\left(t, e_{t}\right)$, where $e_{t}=e(t+s)$ for $s \in[-\tau, 0]$, and function $g\left(t, e_{t}\right)$ is locally bounded. If there exist a differentiable functional $V\left(t, e_{t}\right): R^{+} \times C \rightarrow R^{+}$, and positive constants $\lambda_{1}, \lambda_{2}$ such that, for any $\left(t, e_{t}\right) \in R^{+} \times C$,

$$
\left(\lambda_{1}\|e(t)\|\right)^{2} \leq V\left(t, e_{t}\right),\left.\quad \frac{d V\left(t, e_{t}\right)}{d t}\right|_{(4)} \leq-\delta V\left(t, e_{t}\right)+\lambda_{2} \rho(t),
$$

where $x(t)$ and $y(t)$ are solutions of systems (1.2) and (2.1) respectively, $\delta>0$ and $\rho(t)$ are defined in $\mathbf{H}_{3}$. Then the driver-response systems (1.2) and (2.1) are general decay synchronized in the sense of Definition 2.2, and the convergence rate is $\delta / 2$.

\section{MAIN RESUlTS}

First, under assumption $\mathbf{H}_{3}$, we design the controller $u_{i}(t)$ of response system (2.1) as follows:

$$
u_{i}(t)=-\alpha_{i}(t) \operatorname{sign}\left(e_{i}(t)\right)-\frac{\beta_{i}(t)\|e(t)\| e_{i}(t)}{\|e(t)\|+\rho(t)}, \quad i \in \Pi,
$$

where $\beta_{i}(t)$ and $\alpha_{i}(t)$ for $i \in \Pi$ are control gains satisfying

$$
\begin{aligned}
& E_{i} \triangleq c_{i}^{-}+\beta_{i}^{-}-\sum_{j=1}^{n}\left(\frac{A_{j i}}{\left(1-\zeta_{j i}\right)}+B_{j i} \int_{0}^{\infty} K_{j i}(u) d u+D_{j i}+\tau_{j i} A_{j i}\right)>0 \\
& \alpha_{i}^{-}-\sum_{j=1}^{n}\left(M_{i} d_{j i}^{+}+M_{i} a_{j i}^{+}+O_{i} b_{j i}^{+}\right)>0
\end{aligned}
$$

where $D_{i j}=d_{i j}^{+} H_{j}, A_{i j}=a_{i j}^{+} H_{j}$ and $B_{i j}=b_{i j}^{+} K_{j}$.

Theorem 3.1. Let $\mathbf{H}_{\mathbf{1}}-\mathbf{H}_{\mathbf{3}}$ hold. Then the response network (2.1) can be general decay synchronized with the drive network (1.2) under nonlinear controller (3.1) if the control gains $\beta_{i}(t)$ and $\alpha_{i}(t)$ satisfy inequality (3.2). 
Proof. First, we construct the following Lyapunov-Krasovskii functional

$$
\begin{aligned}
V_{1}(t)= & \sum_{i=1}^{n}\left|e_{i}(t)\right|+\sum_{i=1}^{n} \sum_{j=1}^{n} \int_{t-\tau_{i j}(t)}^{t} \frac{A_{i j}}{\left(1-\zeta_{i j}\right)}\left|e_{j}(s)\right| d s \\
& +\sum_{i=1}^{n} \sum_{j=1}^{n} B_{i j} \int_{0}^{\infty} \int_{t-u}^{t}\left|e_{j}(s)\right| d s d u .
\end{aligned}
$$

Calculating the derivative of $V_{1}(t)$ along system (2.2), we get

$$
\begin{aligned}
\dot{V}_{1}(t)= & \sum_{i=1}^{n} \operatorname{sign}\left(e_{i}(t)\right)\left\{-c_{i}(t) e_{i}(t)+\sum_{j=1}^{n} d_{i j}(t) \tilde{f}_{j}\left(e_{j}(t)\right)+\sum_{j=1}^{n} a_{i j}(t) \tilde{f}_{j}\left(e_{j}\left(t-\tau_{i j}(t)\right)\right)\right. \\
& +\sum_{j=1}^{n} b_{i j}(t) \int_{0}^{\infty} K_{i j}(u) \widetilde{g}_{j}\left(e_{j}(t-u)\right) d u-\alpha_{i}(t) \operatorname{sign}\left(e_{i}(t)\right)-\frac{\beta_{i}(t)\|e(t)\| e_{i}(t)}{\|e(t)\|+\rho(t)\}} \\
& +\sum_{i=1}^{n} \sum_{j=1}^{n} A_{i j}\left(\frac{1}{\left(1-\zeta_{i j}\right)}\left|e_{j}(t)\right|-\frac{\left(1-\dot{\tau}_{i j}(t)\right)}{\left(1-\zeta_{i j}\right)}\left|e_{j}\left(t-\tau_{i j}(t)\right)\right|\right) \\
& +\sum_{i=1}^{n} \sum_{j=1}^{n} B_{i j}\left[\int_{0}^{\infty} K_{i j}(u)\left(\left|e_{j}(t)\right|-\left|e_{j}(t-u)\right|\right) d u d s\right] \\
\leq & \sum_{i=1}^{n}\left\{-c_{i}^{-}\left|e_{i}(t)\right|+\sum_{j=1}^{n} d_{i j}^{+}\left|\widetilde{f}_{j}\left(e_{j}(t)\right)\right|+\sum_{j=1}^{n} a_{i j}^{+}\left|\tilde{f}_{j}\left(e_{j}\left(t-\tau_{i j}(t)\right)\right)\right|\right. \\
& +\sum_{j=1}^{n} b_{i j}^{+} \int_{0}^{\infty} K_{i j}(u)\left|\widetilde{g}_{j}\left(e_{j}(t-u)\right)\right| d u-\alpha_{i}(t)-\frac{\beta_{i}(t)\|e(t)\|\left|e_{i}(t)\right|}{\|e(t)\|+\rho(t)} \\
& +\sum_{j=1}^{n} \frac{A_{i j}}{\left(1-\zeta_{i j}\right)}\left|e_{j}(t)\right|-\sum_{j=1}^{n} A_{i j}\left|e_{j}\left(t-\tau_{i j}(t)\right)\right|+\sum_{j=1}^{n} B_{i j} \int_{0}^{\infty} K_{i j}(u) d u\left|e_{j}(t)\right| \\
& \left.-\sum_{j=1}^{n} B_{i j} \int_{0}^{\infty} K_{i j}(u)\left|e_{j}(t-u)\right| d u\right\} .
\end{aligned}
$$

Now, using $\mathbf{H}_{\mathbf{1}}$, we have

$$
\begin{aligned}
\sum_{i=1}^{n} \sum_{j=1}^{n} d_{i j}^{+}\left|\widetilde{f}_{j}\left(e_{j}(t)\right)\right| & \leq \sum_{i=1}^{n} \sum_{j=1}^{n} d_{i j}^{+}\left(H_{j}\left|e_{j}(t)\right|+M_{j}\right) \\
& =\sum_{i=1}^{n} \sum_{j=1}^{n} D_{i j}\left|e_{j}(t)\right|+\sum_{i=1}^{n} \sum_{j=1}^{n} M_{j} d_{i j}^{+} .
\end{aligned}
$$

Similarly, we have

$$
\begin{aligned}
\sum_{i=1}^{n} \sum_{j=1}^{n} a_{i j}^{+}\left|\widetilde{f}_{j}\left(e_{j}\left(t-\tau_{i j}(t)\right)\right)\right| & \leq \sum_{i=1}^{n} \sum_{j=1}^{n} a_{i j}^{+}\left(H_{j}\left|e_{j}\left(t-\tau_{i j}(t)\right)\right|+M_{j}\right) \\
& =\sum_{i=1}^{n} \sum_{j=1}^{n} A_{i j}\left|e_{j}\left(t-\tau_{i j}(t)\right)\right|+\sum_{i=1}^{n} \sum_{j=1}^{n} a_{i j}^{+} M_{j}
\end{aligned}
$$


and

$$
\begin{aligned}
& \sum_{i=1}^{n} \sum_{j=1}^{n} b_{i j}^{+} \int_{0}^{\infty} K_{i j}(u)\left|\widetilde{g}_{j}\left(e_{j}(t-u)\right)\right| d u \\
& \leq \sum_{i=1}^{n} \sum_{j=1}^{n} B_{i j} \int_{0}^{\infty} K_{i j}(u)\left|e_{j}(t-u)\right| d u+\sum_{i=1}^{n} \sum_{j=1}^{n} b_{i j}^{+} O_{j} .
\end{aligned}
$$

Using $\mathbf{H}_{\mathbf{2}}$, (3.2), (3.4)-(3.7), we have

$$
\begin{aligned}
\dot{V}_{1}(t) \leq & \sum_{i=1}^{n}\left\{-c_{i}^{-}\left|e_{i}(t)\right|+\sum_{j=1}^{n} D_{i j}\left|e_{j}(t)\right|+\sum_{j=1}^{n} M_{j} d_{i j}^{+}+\sum_{j=1}^{n} A_{i j}\left|e_{j}\left(t-\tau_{i j}(t)\right)\right|\right. \\
& +\sum_{j=1}^{n} a_{i j}^{+} M_{j}+\sum_{j=1}^{n} B_{i j} \int_{0}^{\infty} K_{i j}(u)\left|e_{j}(t-u)\right| d u+\sum_{j=1}^{n} b_{i j}^{+} O_{j}-\alpha_{i}(t)-\frac{\beta_{i}(t)|| e(t) \|\left|e_{i}(t)\right|}{\|e(t)\|+\rho(t)} \\
& +\sum_{j=1}^{n} \frac{A_{i j}}{\left(1-\zeta_{i j}\right)}\left|e_{j}(t)\right|-\sum_{j=1}^{n} A_{i j}\left|e_{j}\left(t-\tau_{i j}(t)\right)\right|+\sum_{j=1}^{n} B_{i j} \int_{0}^{\infty} K_{i j}(u) d u\left|e_{j}(t)\right| \\
& \left.-\sum_{j=1}^{n} B_{i j} \int_{0}^{\infty} K_{i j}(u)\left|e_{j}(t-u)\right| d u\right\} \\
\leq & \sum_{i=1}^{n}\left\{-\left[c_{i}^{-}+\beta_{i}^{-}-\sum_{j=1}^{n}\left(\frac{A_{j i}}{\left(1-\zeta_{j i}\right)}+B_{j i} \int_{0}^{\infty} K_{j i}(u) d u+D_{j i}\right)\right]\left|e_{j}(t)\right|\right. \\
& \left.-\left[\alpha_{i}^{-}-\sum_{j=1}^{n}\left(M_{i} d_{j i}^{+}+M_{i} a_{j i}^{+}+O_{i} b_{j i}^{+}\right)\right]+\beta_{i}(t)\left|e_{i}(t)\right|-\frac{\beta_{i}(t)\|e(t)\|\left|e_{i}(t)\right|}{\|e(t)\|+\rho(t)}\right\} \\
\leq & \sum_{i=1}^{n}-\left[c_{i}^{-}+\beta_{i}^{-}-\sum_{j=1}^{n}\left(\frac{A_{j i}}{\left(1-\zeta_{j i}\right)}+B_{j i} \int_{0}^{\infty} K_{j i}(u) d u+D_{j i}\right)\right]\left|e_{j}(t)\right| \\
& +\sum_{i=1}^{n} \frac{\beta_{i}^{+} \rho(t)\left|e_{i}(t)\right|}{\|e(t)\|+\rho(t)} .
\end{aligned}
$$

Next, we construct the following Lyapunov-Krasovskii functional:

$$
V_{2}(t)=\sum_{i=1}^{n} \sum_{j=1}^{n} A_{i j} \int_{-\tau_{i j}}^{0} \int_{t+s}^{t}\left|e_{j}(\theta)\right| d \theta d s
$$

Calculating the derivative of $V_{2}(t)$, we get

$$
\dot{V}_{2}(t)=\sum_{i=1}^{n} \sum_{j=1}^{n}\left[A_{i j}\left(\tau_{i j}\left|e_{j}(t)\right|-\int_{t-\tau_{i j}}^{t}\left|e_{j}(s)\right| d s\right)\right]=\sum_{i=1}^{n} \sum_{j=1}^{n} \tau_{i j} A_{i j}\left|e_{j}(t)\right|-A
$$

where

$$
A=\sum_{i=1}^{n} \sum_{j=1}^{n} A_{i j} \int_{t-\tau_{i j}}^{t}\left|e_{j}(s)\right| d s .
$$

Finally, we construct the following Lyapunov-Krasovskii functional:

$$
V(t)=V_{1}(t)+V_{2}(t)
$$


Then, there exists a scalar $\chi>1$ such that

$$
\sum_{i=1}^{n}\left|e_{i}(t)\right| \leq V(t) \leq \chi \sum_{i=1}^{n}\left|e_{i}(t)\right|+\frac{\chi}{E} A,
$$

where $E=\min _{i \in \Pi}\left\{E_{i}\right\}$. Calculating the derivative of $V(t)$, and using (3.8) and (3.9), we get

$$
\begin{aligned}
\dot{V}(t) \leq & \sum_{i=1}^{n}-\left[c_{i}^{-}+\beta_{i}^{-}-\sum_{j=1}^{n}\left(\frac{A_{j i}}{\left(1-\zeta_{j i}\right)}+B_{j i} \int_{0}^{\infty} K_{j i}(u) d u+D_{j i}+\tau_{j i} A_{j i}\right)\right]\left|e_{j}(t)\right| \\
& +\sum_{i=1}^{n} \frac{\beta_{i}(t) \rho(t)\left\|e_{i}(t)\right\|}{\|e(t)\|+\rho(t)}-A \\
\leq & \sum_{i=1}^{n}-E_{i}\left|e_{i}(t)\right|+\max _{i \in \Pi}\left\{\beta_{i}^{+}\right\} \frac{\|e(t)\| \rho(t)}{\|e(t)\|+\rho(t)}-A
\end{aligned}
$$

Letting $\beta=\max _{i \in \Pi}\left\{\beta_{i}^{+}\right\}>0$, and using the inequality $0 \leq a b /(a+b) \leq a$ for any $a>0, b>0$, we have

$$
\dot{V}(t) \leq \sum_{i=1}^{n}-E_{i}\left|e_{i}(t)\right|+\beta \rho(t)-A .
$$

Taking a small enough $\delta$ such that $\delta \chi<E$, and using (3.10) and (3.12), we get

$$
\begin{aligned}
& \frac{d}{d t} V(t)+\delta V(t) \leq \sum_{i=1}^{n}-E_{i}\left|e_{i}(t)\right|+\beta \rho(t)-A+\delta\left(\chi \sum_{i=1}^{n}\left|e_{i}(t)\right|+\frac{\chi}{E} A\right) \\
& \leq(\delta \chi-E) \sum_{i=1}^{n}\left|e_{i}(t)\right|+\left(\frac{\delta \chi}{E}-1\right) A+\beta \rho(t) \\
& \leq \beta \rho(t),
\end{aligned}
$$

which means that

$$
\dot{V}(t)+\delta V(t) \leq \beta \rho(t) .
$$

Then, from Lemma 2.3, the drive-response systems (1.2) and (2.1) achieve GDS under the adaptive nonlinear controller (3.1). The convergence rate of $e(t)$ approaching zero is $\delta / 2$. The proof is completed.

If, in $\mathbf{H}_{1}, f_{j}(u), g_{j}(u)$ are globally Lipschitz, i.e., $M_{j}=O_{j}=0$, then the $\mathbf{H}_{1}$ becomes

$\mathbf{H}_{1}^{*}: f_{j}(u), g_{j}(u)$ are globally Lipschitz continuous, i.e., there exist constants $H_{j}, K_{j}>0$ such that

$$
\left|f_{j}\left(v_{1}\right)-f_{j}\left(v_{2}\right)\right| \leq H_{j}\left|v_{1}-v_{2}\right|,\left|g_{j}\left(v_{1}\right)-g_{j}\left(v_{2}\right)\right| \leq K_{j}\left|v_{1}-v_{2}\right|,
$$

where $v_{1}, v_{2} \in R$.

In addition, the controller (3.1) in system (1.2) becomes

$$
u_{i}(t)=-\frac{\beta_{i}(t)\|e(t)\| e_{i}(t)}{e_{i}(t)+\rho(t)}, \quad i \in \Pi .
$$

From the proof of Theorem 3.1 and $\mathbf{H}_{1}^{*}$, we have the following result. 
Theorem 3.2. Let $\mathbf{H}_{1}^{*}, \mathbf{H}_{2}, \mathbf{H}_{3}$ hold. Then the response network (2.1) can be general decay synchronized with the drive network (1.2) under the nonlinear controller (3.14) if the control gains $\beta_{i}(t)$ satisfy the following inequality

$$
c_{i}^{-}+\beta_{i}^{-}-\sum_{j=1}^{n}\left(\frac{A_{j i}}{\left(1-\zeta_{j i}\right)}+B_{j i} \int_{0}^{\infty} K_{j i}(u) d u+D_{j i}+\tau_{j i} A_{j i}\right)>0 .
$$

Proof. First, we construct the following Lyapunov-Krasovskii functional:

$$
\begin{aligned}
V_{1}(t)= & \sum_{i=1}^{n}\left|e_{i}(t)\right|+\sum_{i=1}^{n} \sum_{j=1}^{n} \int_{t-\tau_{i j}(t)}^{t} \frac{A_{i j}}{\left(1-\zeta_{i j}\right)}\left|e_{j}(s)\right| d s \\
& +\sum_{i=1}^{n} \sum_{j=1}^{n} B_{i j} \int_{0}^{\infty} \int_{t-u}^{t}\left|e_{j}(s)\right| d s d u .
\end{aligned}
$$

Calculating the derivative of $V_{1}(t)$ along system (2.2), we get

$$
\begin{aligned}
\dot{V}_{1}(t) \leq & \sum_{i=1}^{n}\left\{-c_{i}^{-}\left|e_{i}(t)\right|+\sum_{j=1}^{n} d_{i j}^{+}\left|\widetilde{f}_{j}\left(e_{j}(t)\right)\right|+\sum_{j=1}^{n} a_{i j}^{+}\left|\widetilde{f}_{j}\left(e_{j}\left(t-\tau_{i j}(t)\right)\right)\right|\right. \\
& +\sum_{j=1}^{n} b_{i j}^{+} \int_{0}^{\infty} K_{i j}(u)\left|\widetilde{g}_{j}\left(e_{j}(t-u)\right)\right| d u-\alpha_{i}(t)-\frac{\beta_{i}(t) \| e(t)||\left|e_{i}(t)\right|}{\|e(t)\|+\rho(t)} \\
& +\sum_{j=1}^{n} \frac{A_{i j}}{\left(1-\zeta_{i j}\right)}\left|e_{j}(t)\right|-\sum_{j=1}^{n} A_{i j}\left|e_{j}\left(t-\tau_{i j}(t)\right)\right|+\sum_{j=1}^{n} B_{i j} \int_{0}^{\infty} K_{i j}(u) d u\left|e_{j}(t)\right| \\
& \left.-\sum_{j=1}^{n} B_{i j} \int_{0}^{\infty} K_{i j}(u)\left|e_{j}(t-u)\right| d u\right\} .
\end{aligned}
$$

Now, using $\mathbf{H}_{\mathbf{1}}$, we have

$$
\sum_{i=1}^{n} \sum_{j=1}^{n} d_{i j}^{+}\left|\tilde{f}_{j}\left(e_{j}(t)\right)\right| \leq \sum_{i=1}^{n} \sum_{j=1}^{n} d_{i j}^{+} H_{j}\left|e_{j}(t)\right|=\sum_{i=1}^{n} \sum_{j=1}^{n} D_{i j}\left|e_{j}(t)\right| .
$$

Similarly, we have

$$
\sum_{i=1}^{n} \sum_{j=1}^{n} a_{i j}^{+}\left|\widetilde{f}_{j}\left(e_{j}\left(t-\tau_{i j}(t)\right)\right)\right| \leq \sum_{i=1}^{n} \sum_{j=1}^{n} A_{i j}\left|e_{j}\left(t-\tau_{i j}(t)\right)\right| .
$$

and

$$
\sum_{i=1}^{n} \sum_{j=1}^{n} b_{i j}^{+} \int_{0}^{\infty} K_{i j}(u)\left|\widetilde{g}_{j}\left(e_{j}(t-u)\right)\right| d u \leq \sum_{i=1}^{n} \sum_{j=1}^{n} B_{i j} \int_{0}^{\infty} K_{i j}(u)\left|e_{j}(t-u)\right| d u .
$$

Using $\mathbf{H}_{\mathbf{2}}$, and (3.14)-(3.19), we have

$$
\begin{aligned}
\dot{V}_{1}(t) \leq & \sum_{i=1}^{n}-\left[c_{i}^{-}+\beta_{i}^{-}-\sum_{j=1}^{n}\left(\frac{A_{j i}}{\left(1-\zeta_{j i}\right)}+B_{j i} \int_{0}^{\infty} K_{j i}(u) d u+D_{j i}\right)\right]\left|e_{j}(t)\right| \\
& +\sum_{i=1}^{n} \frac{\beta_{i}^{+} \rho(t)\left|e_{i}(t)\right|}{\|e(t)\|+\rho(t)}
\end{aligned}
$$


Next, we construct the following Lyapunov-Krasovskii functional:

$$
V_{2}(t)=\sum_{i=1}^{n} \sum_{j=1}^{n} A_{i j} \int_{-\tau_{i j}}^{0} \int_{t+s}^{t}\left|e_{j}(\theta)\right| d \theta d s
$$

Calculating the derivative of $V_{2}(t)$, we get

$$
\dot{V}_{2}(t)=\sum_{i=1}^{n} \sum_{j=1}^{n}\left[A_{i j}\left(\tau_{i j}\left|e_{j}(t)\right|-\int_{t-\tau_{i j}}^{t}\left|e_{j}(s)\right| d s\right)\right]=\sum_{i=1}^{n} \sum_{j=1}^{n} \tau_{i j} A_{i j}\left|e_{j}(t)\right|-A,
$$

where

$$
A=\sum_{i=1}^{n} \sum_{j=1}^{n} A_{i j} \int_{t-\tau_{i j}}^{t}\left|e_{j}(s)\right| d s .
$$

Finally, we construct the following Lyapunov-Krasovskii functional $V(t)=V_{1}(t)+V_{2}(t)$. Then, there exists a scalar $\chi>1$ such that

$$
\sum_{i=1}^{n}\left|e_{i}(t)\right| \leq V(t) \leq \chi \sum_{i=1}^{n}\left|e_{i}(t)\right|+\frac{\chi}{E} A,
$$

where $E=\min _{i \in \Pi}\left\{E_{i}\right\}$. Calculating the derivative of $V(t)$, and using (3.20)-(3.21), we get

$$
\begin{aligned}
\dot{V}(t) \leq & \sum_{i=1}^{n}-\left[c_{i}^{-}+\beta_{i}^{-}-\sum_{j=1}^{n}\left(\frac{A_{j i}}{\left(1-\zeta_{j i}\right)}+B_{j i} \int_{0}^{\infty} K_{j i}(u) d u+D_{j i}+\tau_{j i} A_{j i}\right)\right]\left|e_{j}(t)\right| \\
& +\sum_{i=1}^{n} \frac{\beta_{i}(t) \rho(t)\left\|e_{i}(t)\right\|}{\|e(t)\|+\rho(t)}-A \\
\leq & \sum_{i=1}^{n}-E_{i}\left|e_{i}(t)\right|+\max _{i \in \Pi}\left\{\beta_{i}^{+}\right\} \frac{\|e(t)\| \rho(t)}{\|e(t)\|+\rho(t)}-A .
\end{aligned}
$$

Letting $\beta=\max _{i \in \Pi}\left\{\beta_{i}^{+}\right\}>0$ and using the inequality $0 \leq a b /(a+b) \leq a$ for any $a>0, b>0$, we have

$$
\dot{V}(t) \leq \sum_{i=1}^{n}-E_{i}\left|e_{i}(t)\right|+\beta \rho(t)-A .
$$

Now taking a small enough $\delta$ such that $\delta \chi<E$, we conclude from (3.22) and (3.24) that

$$
\begin{aligned}
& \frac{d}{d t} V(t)+\delta V(t) \leq \sum_{i=1}^{n}-E_{i}\left|e_{i}(t)\right|+\beta \rho(t)-A+\delta\left(\chi \sum_{i=1}^{n}\left|e_{i}(t)\right|+\frac{\chi}{E} A\right) \\
& \leq(\delta \chi-E) \sum_{i=1}^{n}\left|e_{i}(t)\right|+\left(\frac{\delta \chi}{E}-1\right) A+\beta \rho(t) \\
& \leq \beta \rho(t),
\end{aligned}
$$

which means that $\dot{V}(t)+\delta V(t) \leq \beta \rho(t)$. Then, from Lemma 2.3, the drive-response systems (1.2) and (2.1) achieve GDS under the adaptive nonlinear controller (3.14). The convergence rate of $e(t)$ approaching zero is $\delta / 2$. The proof is completed.

Remark 3.3. [25] The function $\psi$ was used as the decay function. so, the $\psi$-type stability is also said to be stability with general decay rate. If $\psi(t)=e^{\alpha t}, \psi(t)=(1+t)^{\alpha}$ and $\psi(t)=$ $1+\alpha \log (1+t)$ for any $\alpha>0$, then the $\psi$-type stability can be specialized as exponential synchronization, polynomial synchronization and logarithmic synchronization, respectively. In 
this paper, if $\rho(t)=0$, which is given in controller (3.1), then the GDS can be specialized as exponential synchronization.

\section{NUMERiCAL Simulations}

In this section we give one example to illustrate the results obtained in this paper.

Example 4.1. For $n=2$, we consider the following delayed cellular neural networks with time-varying coefficients

$$
\begin{aligned}
\dot{x}_{i}(t)= & -c_{i}(t) x_{i}(t)+\sum_{j=1}^{2} d_{i j}(t) f_{j}\left(x_{j}(t)\right)+\sum_{j=1}^{2} a_{i j}(t) f_{j}\left(x_{j}\left(t-\tau_{i j}(t)\right)\right) \\
& +\sum_{j=1}^{2} b_{i j}(t) \int_{0}^{\infty} K_{i j}(u) g_{j}\left(x_{j}(t-u)\right) d u+I_{i}(t)
\end{aligned}
$$

where $f_{1}(x)=f_{2}(x)=\tanh (x), g_{1}(t)=g_{2}(t)=\tanh (x)-\sin (x)$ and

$$
\begin{aligned}
& {\left[\begin{array}{l}
c_{1}(t) \\
c_{2}(t)
\end{array}\right]=\left[\begin{array}{c}
\frac{11+\sin (t)}{10} \\
\frac{115+15 \sin (t)}{100}
\end{array}\right],\left[\begin{array}{ll}
d_{11}(t) & d_{12}(t) \\
d_{21}(t) & d_{22}(t)
\end{array}\right]=\left[\begin{array}{cc}
2+0.01 \sin (t) & -0.11+0.001 \sin (t) \\
-2.5+0.01 \sin (t) & 3.5+0.01 \sin (t)
\end{array}\right],} \\
& {\left[\begin{array}{l}
I_{1}(t) \\
I_{2}(t)
\end{array}\right]=\left[\begin{array}{c}
\frac{1+\sin (t)}{1000} \\
\frac{2+1.5 \sin (t)}{1000}
\end{array}\right],\left[\begin{array}{ll}
a_{11}(t) & a_{12}(t) \\
a_{21}(t) & a_{22}(t)
\end{array}\right]=\left[\begin{array}{cc}
-1.6+0.001 \sin (t) & -0.1+0.001 \sin (t) \\
-0.18+0.001 \sin (t) & -2.4+0.01 \sin (t)
\end{array}\right],} \\
& {\left[\begin{array}{ll}
K_{11}(u) & K_{12}(u) \\
K_{21}(u) & K_{22}(u)
\end{array}\right]=\left[\begin{array}{ll}
e^{u} & e^{u} \\
e^{u} & e^{u}
\end{array}\right],\left[\begin{array}{ll}
\tau_{11}(t) & \tau_{12}(t) \\
\tau_{21}(t) & \tau_{22}(t)
\end{array}\right]=\left[\begin{array}{cc}
\frac{e^{t}}{1.5+e^{t}} & \frac{e^{t}}{1.4+e^{t}} \\
\frac{e^{t}}{1.4+e^{t}} & \frac{e^{t}}{1.5+e^{t}}
\end{array}\right],} \\
& {\left[\begin{array}{ll}
b_{11}(t) & b_{12}(t) \\
b_{21}(t) & b_{22}(t)
\end{array}\right]=\left[\begin{array}{cc}
0.2+0.01 \sin (t) & 0.01+0.001 \sin (t) \\
-0.2+0.001 \sin (t) & 0.16+0.01 \sin (t)
\end{array}\right] \text {. }}
\end{aligned}
$$

The corresponding response system is described by

$$
\begin{aligned}
\dot{y}_{i}(t)= & -c_{i}(t) y_{i}(t)+\sum_{j=1}^{2} d_{i j}(t) f_{j}\left(y_{j}(t)\right)+\sum_{j=1}^{2} a_{i j}(t) f_{j}\left(y_{j}\left(t-\tau_{i j}(t)\right)\right) \\
& +\sum_{j=1}^{2} b_{i j}(t) \int_{0}^{\infty} K_{i j}(u) g_{j}\left(y_{j}(t-u)\right) d u+I_{i}(t)+u_{i}(t)
\end{aligned}
$$

where $c_{i}(t), a_{i j}(t), b_{i j}(t), d_{i j}(t), f_{j}(t), g_{j}(t), \tau_{i j}(t)$ and $I_{i}(t)$ are the same as in system (4.1).

The numerical simulations of the system (4.1) and the system (4.2) with initial values $x_{1}(s)=$ $0.2, x_{2}(s)=0.5$ and $y_{1}(s)=-1.3, y_{2}(s)=2.1$ for $s \in[-1,0]$ are represented in

From Fig.4.1 and Fig.4.2, we can see that system (4.1) and system (4.2) have chaotic attractors. 

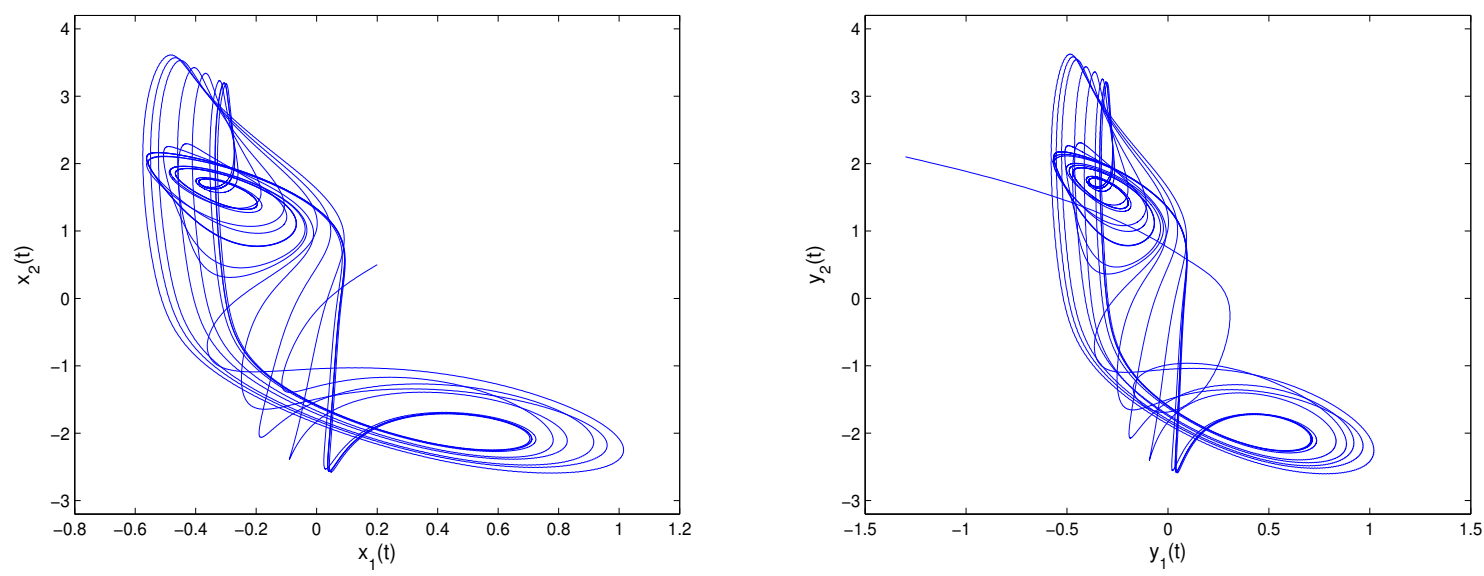

Fig. 4.1. The chaotic behavior of delayed cellular neural network system (4.1) and (4.2).

The nonlinear controller $u_{i}(t)$ is designed as follows

$$
u_{i}(t)=-\alpha_{i}(t) \operatorname{sign}\left(e_{i}(t)\right)-\frac{\beta_{i}(t)\|e(t)\| e_{i}(t)}{\|e(t)\|+\rho(t)}, \quad i \in \Pi,
$$

where $e_{i}(t)=y_{i}(t)-x_{i}(t)$ for $i=1,2$.

It is easy to estimate that $H_{j}=K_{j}=1, M_{j}=0.045, O_{j}=0.035$ and $\tau_{i j}=1$. Thus, the assumptions $\mathbf{H}_{1}$ and $\mathbf{H}_{2}$ are satisfied. Let $\rho(t)=e^{-0.1 t}$ and choose $\alpha_{1}^{-}=0.5, \alpha_{2}^{-}=0.6$, $\beta_{1}^{-}=7.5, \beta_{2}^{-}=6$. Then, the assumption $\mathbf{H}_{3}$ and the inequality (3.2) of Theorem 3.1 are satisfied. Therefore, according to Theorem 3.1, the drive-response systems (4.1) and (4.2) can be achieved by GDS under the controller (4.3). The time evolution of synchronization errors between systems (4.1) and (4.2) are demonstrated in Fig.4.2. The synchronization curves between systems (4.1) and (4.2) are shown in Fig.4.3.

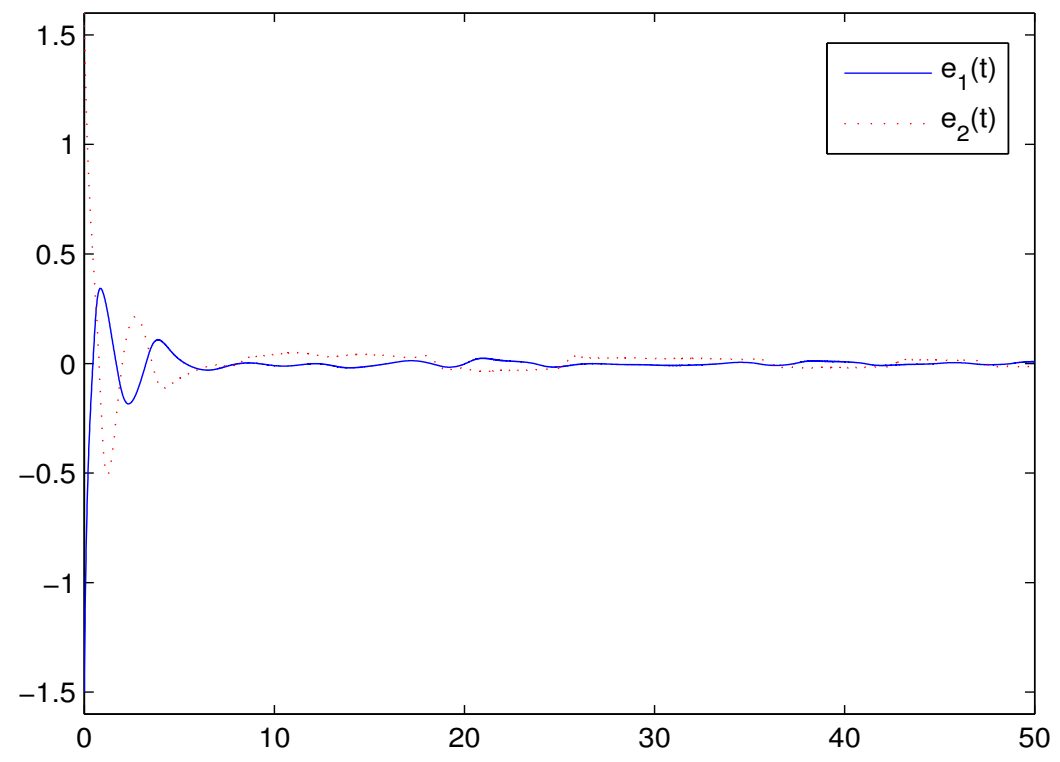

Fig.4.2. The evaluation of synchronization error $e_{1}(t)$ and $e_{2}(t)$. 

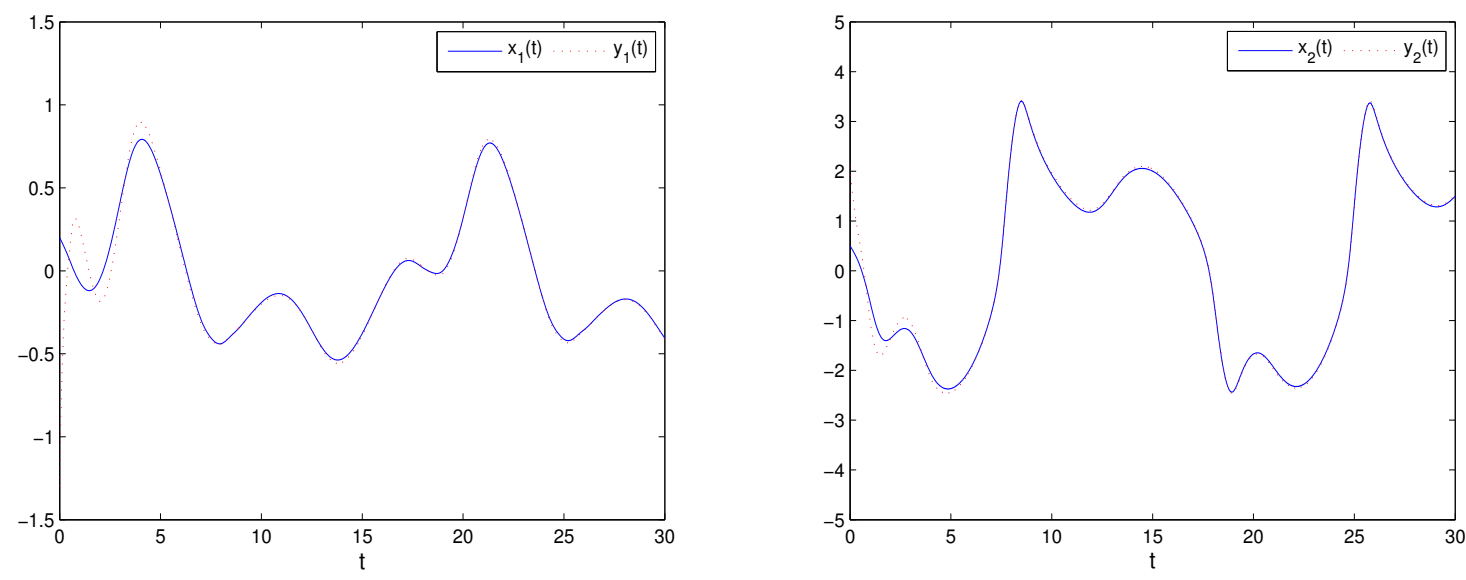

Fig.4.3. Synchronization curves of $x_{1}(t), y_{1}(t)$ and $x_{2}(t), y_{2}(t)$.

\section{THE CONCLUSION}

In the present analysis, to the best knowledge of the authors, this is the first result on the GDS problem for a class of general non-autonomous cellular neural networks with time variable delays and infinite delays. By constructing suitable Lyapunov-Krasovskii functionals and applying the method given in $[24,25]$, we obtained some new sufficient conditions on the general decay synchronization of the drive-response systems (1.2) and (2.1). In addition, an example and its numerical simulations are provided to validate the correctness of the theoretical results in this paper. In comparison to previous results presented in [21]-[28], results of this paper are less conservative and more general. Moreover, the theoretical results in this paper can be seen as a complement and an extension to the previous works [21]-[28].

\section{Funding}

This work was supported by the National Natural Science Foundation of China under Grant Nos. 11662020 and 61662077.

\section{REFERENCES}

[1] C. Ke, Y. He, M. Wu, Exponential synchronization of neural networks with time-varying mixed delays and sampled-data, Neurocomputing 74 (2010), 265-273.

[2] A. Muhammadhaji, A. Abdurahman, H. Jiang, Finite-time synchronization of complex dynamical networks with time-varying delays and nonidentical nodes, J. Control Sci. Eng. 2017 (2017), Article ID 5072308.

[3] T. Li, A. Song, S. Fei, Y. Guo, Synchronization control of chaotic neural networks with time-varying and distributed delays, Nonlinear Anal. 71 (2009), 2372-2384.

[4] W. He, F. Qian, J. Cao, Pinning-controlled synchronization of delayed neural networks with distributed-delay coupling via impulsive control, Neural Networks 85 (2017), 1-9.

[5] J. Cao, J. Wang, Global exponential stability and periodicity of recurrent neural networks with time delays, IEEE Trans. Circuits Syst. 52 (2005), 920-931.

[6] R. Rifhat, A. Muhammadhaji, Z. Teng, Global Mittag-Leffler synchronization for impulsive fractional-order neural networks with delays, Int. J. Nonlinear Sci. Numer. 19 (2018), 205-213.

[7] D. Wang, L. Huang, L. Tang, Dissipativity and synchronization of generalized BAM neural networks with multivariate discontinuous activations, IEEE Trans. Neural Netw. Learning Syst. 29 (2018), 3815-3827.

[8] K. Shi, X. Liu, Y. Tang, H. Zhu, S. Zhong, Some novel approaches on state estimation of delayed neural networks, Info. Sci. 372 (2016),313-331. 
[9] K. Shi, J. Wang, Y. Tang, S. Zhong, Reliable asynchronous sampled-data filtering of T-S fuzzy uncertain delayed neural networks with stochastic switched topologies, Fuzzy Sets and Systems 381 (2020),1-25.

[10] K. Shi,J. Wang, Y. Tang, S. Zhong,J. Cheng, Non-fragile memory filtering of T-S fuzzy delayed neural networks based on switched fuzzy sampled-data control, Fuzzy Sets and Systems, 394 (2020),40-64

[11] J. Wang, H. Wu, T. Huang, S. Ren, Pinning control strategies for synchronization of linearly coupled neural networks with reaction-diffusion terms, IEEE Trans. Neural Netw. Learn. Syst. 27 (2016), 749-761.

[12] H. Zhao,L. Li,H. Peng,J. Xiao,Y. Yang, M. Zheng, Impulsive control for synchronization and parameters identification of uncertain multi-links complex network, Nonlinear Dyn. 83 (2016), 1437-1451.

[13] A. Abdurahman, H. Jiang, Z. Teng, Finite-time synchronization for fuzzy cellular neural networks with timevarying delays, Fuzzy Sets and Systems, 297 (2016), 96-111.

[14] J. Cao, R. Li, Fixed-time synchronization of delayed memristor-based recurrent neural networks, Sci. China Inf. Sci. 60 (2017), 104-118.

[15] Z. Zhang, A. Li, S. Yu, Finite-time synchronization for delayed complex-valued neural networks via integrating inequality method, Neurocomputing 318 (2018), 248-260.

[16] Z. Zhang, L. Ren, New sufficient conditions on global asymptotic synchronization of inertial delayed neural networks by using integrating inequality techniques, Nonlinear Dyn. 95 (2019),905-917.

[17] S. Wen, Z. Zeng, T. Huang,Y. Chen, Passivity analysis of memristor-based recurrent neural networks with time-varying delays, J. Frankl. Inst. 350 (2013), 2354-2370.

[18] A. Wu, Z. Zeng, X. Zhu, J. Zhang, Exponential synchronization of memristor-based recurrent neural networks with time delays, Neurocomputing 74 (2011), 3043-3050.

[19] J. Wang, K. Shi, Q. Huang, S. Zhong, D. Zhang, Stochastic switched sampled-data control for synchronization of delayed chaotic neural networks with packet dropout, Appl. Math.Comput. 335 (2018), 211-230.

[20] K. Shi, Y. Tang, X. Liu, S. Zhong, Non-fragile sampled-data robust synchronization of uncertain delayed chaotic Lurie systems with randomly occurring controller gain fluctuation, ISA Trans. 66 (2017), 185-199.

[21] A. Muhammadhaji, Z Teng, General decay synchronization for recurrent neural networks with mixed time delays, J. Syst. Sci. Complex. 33 (2020), 672-684.

[22] A. Muhammadhaji, A. Abdurahman, General decay synchronization for fuzzy cellular neural networks with time-varying delays, Int. J. Nonlin. Sci. Numer. 20 (2019), 551-560.

[23] M. Sader, A. Abdurahman, H. Jiang, General decay lag synchronization for competitive neural networks with constant delays, Neural Process. Lett. 50 (2019), 445-457.

[24] L. Wang, Y. Shen, G. Zhang, Synchronization of a class of switched neural networks with time-varying delays via nonlinear feedback control, IEEE Trans. Cyber. 46 (2016), 2300-2310.

[25] L. Wang, Y. Shen, G. Zhang, General decay synchronization stability for a class of delayed chaotic neural networks with discontinuous activations, Neurocomputing 179 (2016), 169-175.

[26] A. Muhammadhaji, A. Halik, Synchronization stability for recurrent neural networks with time-varying delays, ScienceAsia, 45 (2019), 179-186.

[27] Y. Tang, Exponential convergence of delayed cellular neural networks with time-varying coefficients, Appl. Math. Lett. 21 (2008), 872-876.

[28] C. Xu,P. Li, New proof on exponential convergence for cellular neural networks with time-varying delays, Boundary Value Probl. 2019 (2019), 123. 